\title{
Addressed Fiber Bragg Sensors for Vibration Diagnostics of Complex Objects
}

\author{
A. Kuznetsov ${ }^{1, *}$, K. Lipatnikov ${ }^{1,}$, A. Sakhabutdinov ${ }^{1,}$, O. Morozovi, K. Karimovi,
}

1 Department of Radiophotonics and Microwave Technologies, Kazan National Research Technical University named after A.N. Tupolev-KAI, 420111 Kazan, Russia; AAKuznetsov@kai.ru (AK), KALipatnikov@kai.ru (KL), kazanboy@yandex.ru (AS), microoil@mail.ru (OM), mail12kamil2000@mail.ru (KK)

* Correspondence: AAKuznetsov@kai.ru

\begin{abstract}
The paper presents the results of a study of the concept of address fiber Bragg structures in the problem of vibration control. The mathematical model of measuring transformation is presented; the experimental study of a vibration diagnostics system based on Address Fiber Bragg Gratings is carried out; the quantitative and qualitative comparative assessment with electronic accelerometers is made; the gain by an order of magnitude in some parameters is shown.
\end{abstract}

Keywords: Fiber Bragg Structure, microwave photonics, vibration, fiber optic sensor, Address Fiber Bragg Grating.

\section{Introduction}

The problem of predictive control of modern technically complex objects condition is acute. The widespread transition to the operation of equipment "on condition" requires built-in control systems. A special place here is occupied by vibration monitoring systems. The essence of this technique is to measure the spectrum of natural (or forced) oscillations of the object under study in a wide frequency band. Based on the available statistical data, the spectrum harmonics are analyzed, on the basis of which conclusions are drawn about the presence of defects in the nodes of the system. Most often, this method does not diagnose the exact location of a defect in a complex system (since this requires colossal amounts of statistical data for each type of defects and its various combinations), but only indicates the presence of a problem (with some degree of "criticality") and the need delivery to inspect the product.

Currently, there is a wide range of electrical vibration sensors with good sensitivity and frequency range, work is underway to create similar systems using fiber-optic sensors. The main advantages of optical sensors in comparison with electrical ones are well known, for this task they are especially important: immunity of the sensitive element to electromagnetic fields (since often vibration diagnostic systems are installed on highpower electric machines that are sources of strong magnetic fields); small size and weight (which makes it possible to abandon the strapping in the form of a sensor and embed the sensitive elements directly into the design of the product); ease of combining a large number of sensors into groups.

Let's consider the main types of optical vibration sensors [1]. They can be divided into three large classes: sensors based on intensity modulation, interferometric (based on a Fabry-Perot interferometer), and based on fiber Bragg gratings (FBGs). In this paper, we do not consider distributed vibration sensors. 


\section{Varieties of Optical Vibration Sensors}

\subsection{Sensors Based on Intensity Modulation}

The sensors based on intensity modulation have perhaps the longest history since the mid-1980s.

The principle of operation of such sensors is to register the change in the power of the optical signal passing through the sensor because of the influence of the investigated mechanical vibration. The changing in power can be obtained by, for example, [2-10]: mutual movement of the transmitting and receiving fibers, introducing losses through a series of micro bends, or using a moving reflector/opaque screen. One of the variations in the implementation of the latter method is the use of a test object as a reflective surface (non-contact sensor, Figure 1).

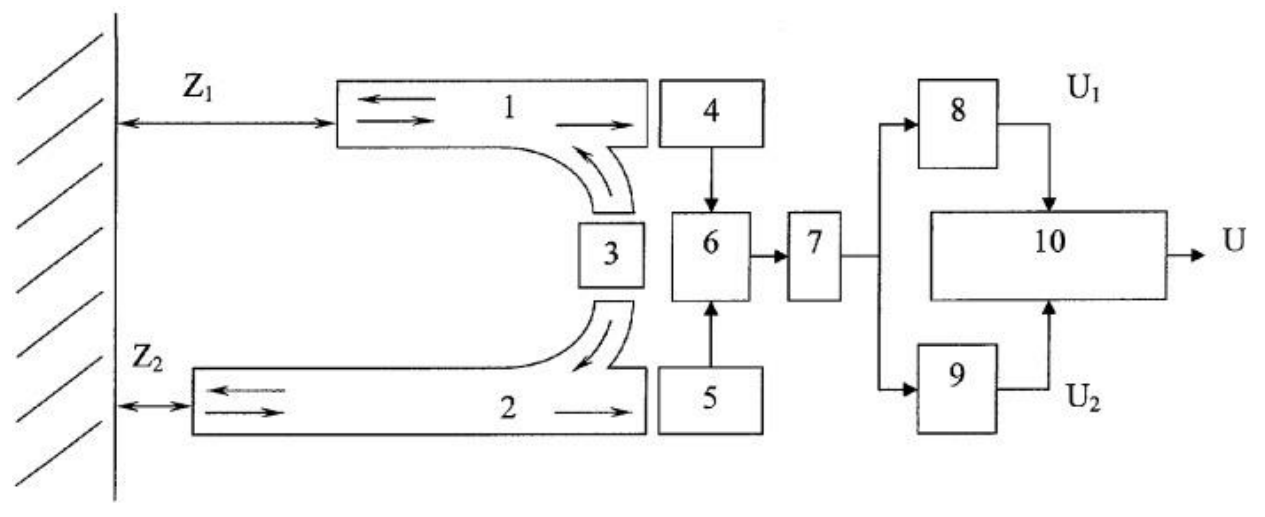

Figure 1. Block diagram of a non-contact vibration sensor [11]. 1, 2- optical fibers, 3 - light source, 4, 5 - optical modulators, 6 - photodetector, 7 - amplifier, 8, 9 - filters, 10 - processor; Z1, Z2 distances between fiber ends and object under control, U1, U2 - informational signals of two arms, $\mathrm{U}$ - calculated signal.

This approach minimizes the influence of the design of the sensor itself on the measurements (natural frequencies of the mechanical system of the sensor and its mass). However, a common disadvantage of an intensity modulation-based sensor is its low accuracy (since information about the measured parameter is embedded in the amplitude change, which requires a good SNR). An increase in accuracy can be obtained by converting the measurements to the spectral region. The interferometric sensors are built on this principle.

Such sensors most often use optical fibers operating in the visible part of the spectrum and having a large diameter of the light-guiding core, which reduces the requirements for the accuracy of their alignment.

\subsection{Interferometric Sensors}

In sensors of this type, a Fabry-Perot interferometer is most often used, which consists of two plane-parallel reflecting surfaces, one of which is semitransparent (through which optical radiation is input and output). Structurally, such sensors are a special case of sensors of the previous type, however, their key difference is that information is embedded in the signal spectrum, which can significantly increase the sensitivity and resolution. The generalized design of such a sensor and interrogation device is shown in Figure 2. The principle of operation of the sensor is based on the transformation of mechanical vibrations of the object under study into a change in the characteristics of the interferometer (free spectral range), the optoelectronic circuit measures the spectral characteristics of the filter and converts these values into the desired amplitude and frequency of vibration. 


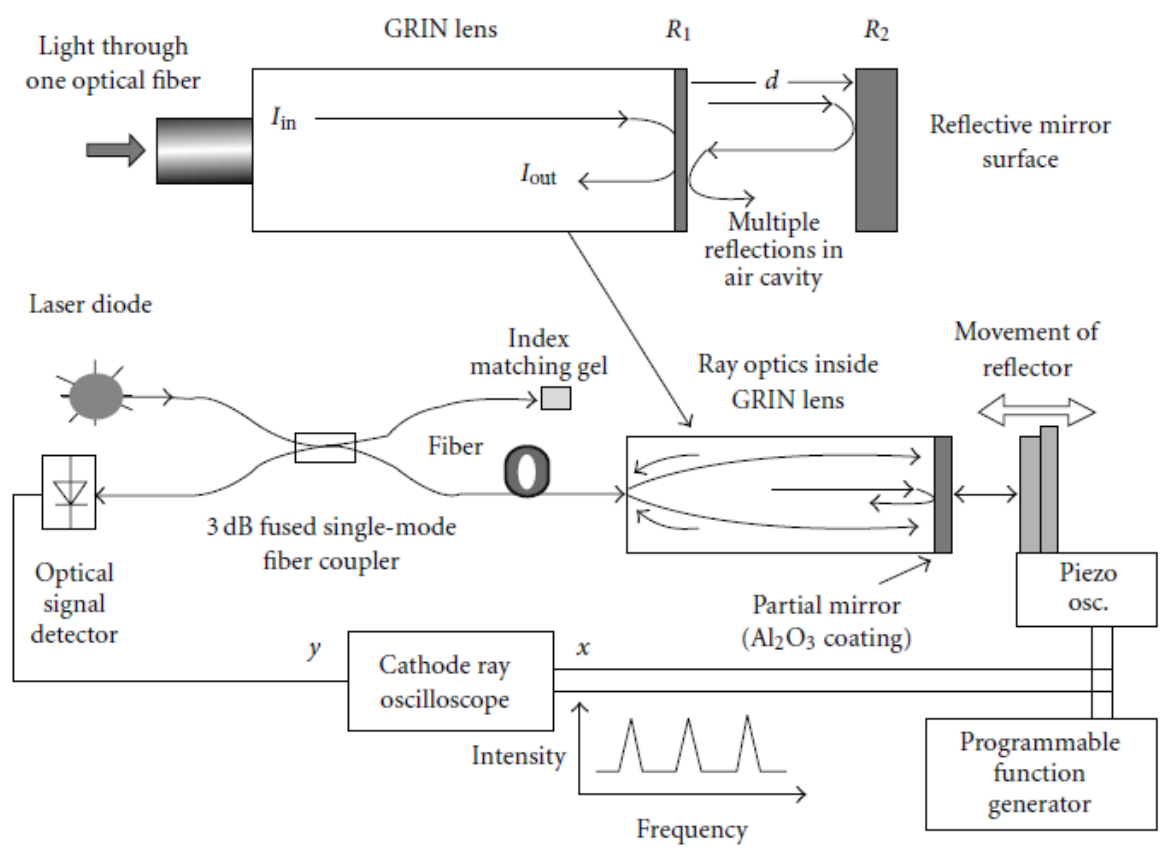

Figure 2. Vibration sensor based on a Fabry-Perot interferometer [12].

As a key disadvantage, it is worth noting the high requirements for the production technology of such sensors.

\subsection{Fiber Bragg Grating Sensors}

Currently, the most rapid growth is observed in optical sensors based on fiber Bragg gratings. The key advantage over interferometric sensors is the all-fiber design of the sensing element, which simplifies fabrication and increases reliability. The shift of the FBG's wavelength, depending on the applied axial deformation and temperature, is described as follows:

$$
\Delta \lambda=2 \cdot n_{e f f} \cdot \Lambda \cdot\left[1-\frac{n_{e f f}}{2} \cdot\left(P_{12}-v \cdot\left(P_{11}+P_{12}\right)\right) \cdot \Delta \varepsilon+\left(\alpha+n_{e f f}{ }^{-1} \cdot \frac{d n_{e f f}}{d T}\right) \cdot \Delta T\right]
$$

where $\Delta T$ - temperature change, $\Delta \varepsilon$ - applied axial deformation (tension), $P_{m n}-$ Pockels coefficients of the elastic-optical tensor, $v$ - Poisson's ratio, $\alpha$-thermal expansion coefficient of quartz glass, $n_{e f f}$ - effective refractive index of the fundamental mode, $\Delta \lambda$ - shift of the FBG's wavelength, $\Lambda$ - period of FBG.

The principle of operation of vibration sensors based on FBG is to convert the energy of forced vibrations of the inertial mass into deformation of the Bragg grating, as a result, the deviation of the grating wavelength will reflect the spectrum of mechanical vibrations acting on it [13-15]. In order to compensate for the temperature sensitivity of FBGs, differential schemes are used [16-17], in which the response of two FBGs to vibration is in antiphase, which allows subtracting the slowly changing wavelength shift from temperature (Figure 3).

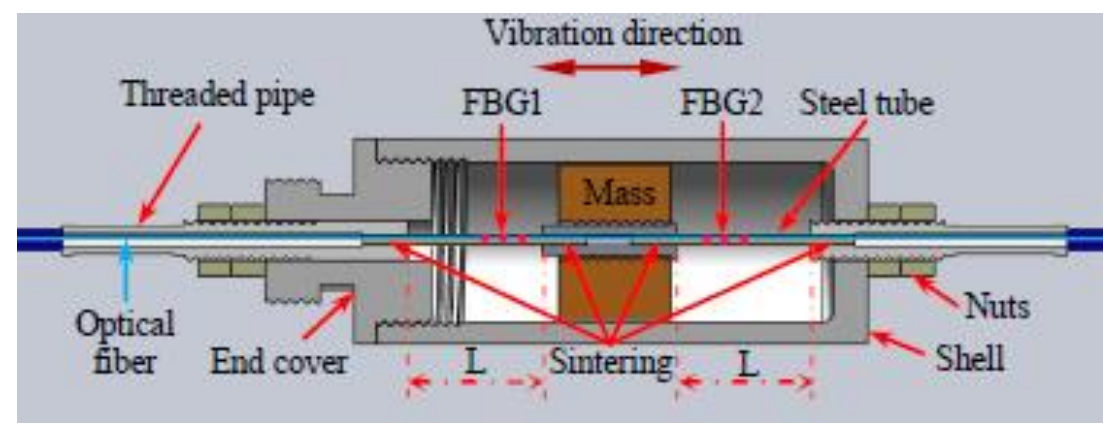


Figure 3. FBG-based differential vibration sensor [16].

To register signals from sensors, special devices are used - interrogators, which are a spectrometer with a combined radiation source. Depending on the construction principle of the device, the cutoff frequency of scanning can be $\sim 100 \mathrm{~Hz}$ (interrogators on tunable filters) or units of $\mathrm{kHz}$ (interrogators with a spectrometer on a CCD array). It can be seen that the boundary detected vibration frequency does not exceed a few $\mathrm{kHz}$ (two times lower than the scanning frequency), this circumstance limits the scope of application of the sensors. There are approaches to the construction of polling systems, where the frequency of information retrieval is much higher [18-19]. The disadvantage of this approach is the complexity of the multiplexing of the sensors.

In our work, we propose to use addressed fiber Bragg structures (AFBG) as sensing elements. The AFBG theory was first proposed and developed within the framework of the scientific school of the authors, a series of technical solutions for temperature and static deformation control [20-22] are proposed. The use of address structures makes it possible to get rid of traditional interrogators, which makes it possible to significantly expand the range of detected frequencies while retaining one of the main advantages of FBG sensors - the simplicity of multiplexing. However, to date, the problem of using such structures to control dynamic processes in a wide range of exposure frequencies remains unsolved. Our study is devoted to solving this problem.

\section{Modeling the AFBG Measurement Conversion for Vibration Control}

A typical diagram of the measuring system based on the through-type AFBG is shown in Figure 4.

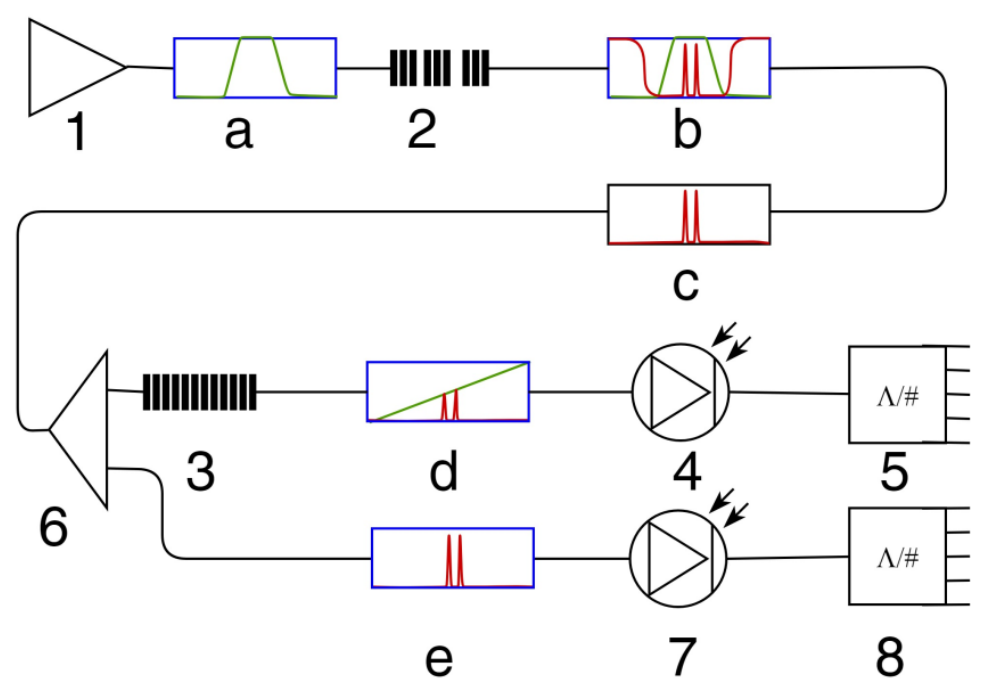

Figure 4. Interrogation system of one AFBG-sensor: (1) - superluminescent light source, (2) AFBG-sensor, (3) - frequency-amplitude conversion optical dispersive element, (4) - measuring channel photodetector, (5) - measuring channel processing unit, (6) - optical splitter, (7) - reference channel photodetector, (8) - reference channel processing unit; (a) - superluminescent source emission spectrum, (b) и (с) - output spectrum of AFBG-sensor, (d) - output spectrum of dispersive element, (e) - reference channel spectrum.

At the output of the photodetector, an amplitude-modulated signal is formed, in which the address frequency $\omega_{A F B G}$ acts as a carrier, and the desired vibration $E_{v i b}(t)$ acts as an envelope:

$$
U_{P D}(t)=U_{0} \cdot\left(1+k \cdot \frac{E_{v i b}(t)}{\left|E_{v i b}(t)\right|_{\max }}\right) \cdot \cos \left(\omega_{A F B G} \cdot t\right),
$$


where $U_{P D}(t)$ - output signal of photodetector, $U_{0}$ - amplitude, $k$ - modulation depth, $E_{v i b}(t)$ - desired vibration, $\left|E_{v i b}(t)\right|_{\max }$ - maximum value of $E_{v i b}(t), \omega_{A F B G}$ - address frequency.

The parameter $k$ (modulation depth) in Equation (2) characterizes the transfer characteristic of the mechanical part of the sensor and the dispersion element. The condition $k \leq 1$ must be met, otherwise overmodulation will occur and the desired signal will be distorted. By detecting the low-frequency envelope, the shape of the desired vibration effect can be restored.

Let's get a mathematical model of the measuring conversion. Let us write the harmonics of the optical spectrum that form the address bias frequency $\omega_{A F B G}$ in the form:

$$
\begin{aligned}
& L(t)=a_{L} \cdot \Omega(t) \cdot \cos \left[\left(\omega-\omega_{A F B G} / 2\right) \cdot t\right], \\
& R(t)=a_{R} \cdot \Omega(t) \cdot \cos \left[\left(\omega+\omega_{A F B G} / 2\right) \cdot t\right] .
\end{aligned}
$$

where $\Omega, a_{L}$ and $a_{R}$ are the sought vibration parameters (frequency and amplitude).

In Equations (3) and (4), the sought vibration effect $\Omega(t)$ is reflected in the change in the amplitude of the left and right spectral components.

The resulting bias signal on photodetector is defined as:

$$
P D(t)=(L(t)+R(t))^{2},
$$

After simplification (harmonics with frequencies by several orders of magnitude higher than the passband of the photodetector from Equation (5) are excluded), we obtain:

$$
P D(t) \equiv A_{L}(t)^{2}+A_{R}(t)^{2}+2 \cdot A_{L}(t) \cdot A_{R}(t) * \cos \left(\omega_{A F B G} \cdot t\right),
$$

The envelope signal in Equation (6) reflects $\cos [\Omega \cdot t]$ through a certain proportionality coefficient determined by the transfer characteristics of the mechanical part of the sensor and the frequency response of the dispersion element. It is advisable to clarify the value of this coefficient at the stage of calibration of the measuring system.

Let's simulate the received measurement transformation. We put the following initial data $(\mathrm{au}): a_{L}=a_{R}=1, \omega_{A F B G}=100, \Omega(t)=A_{v 1} \cdot \cos \left(\Omega_{01} \cdot t\right)+A_{v 2} \cdot \cos \left(\Omega_{02} \cdot t\right), \Omega_{01}=$ $1, \Omega_{02}=0.3$. The signal from the output of the photodetector $P D(t)$ will look as follows (Figure 5):

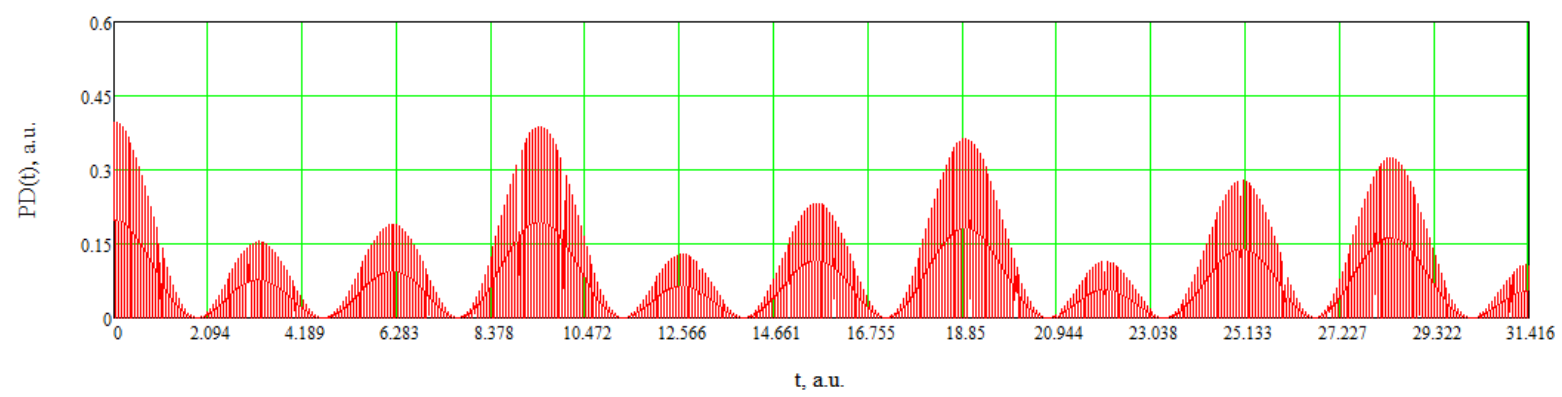

Figure 5. Signal from the photodetector output.

The spectrum of this signal is shown in Figure 6,a, and the envelope spectrum is shown in Figure 6,b. 


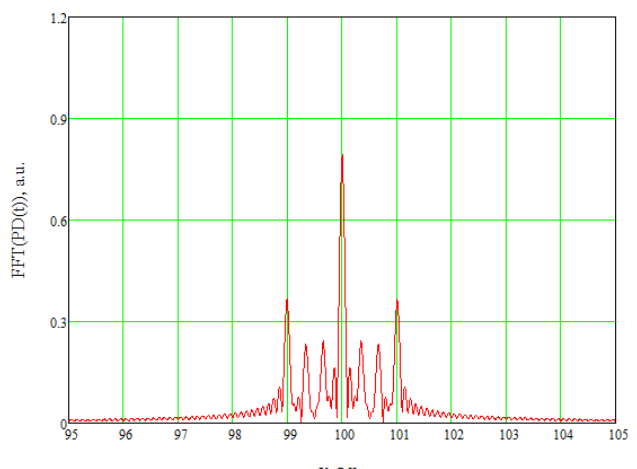

(a)

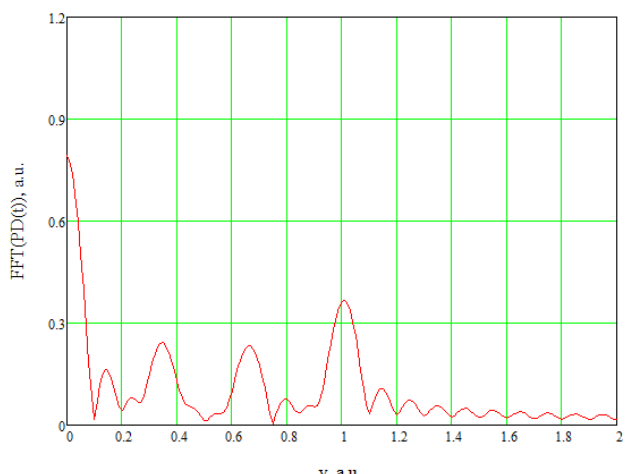

(b)

Figure 6. The spectrum of the signal under study at the output of the photodetector (a) and its envelope (b).

The signal in Figure 6,a-spectrum of an amplitude-modulated signal, with a carrier frequency $\omega_{A F B G}=100 a$. $u$, the envelope (Figure 6,b) contains the combination frequencies of the desired vibration action

\section{Experiment and Discussion}

The developed model of the system was investigated. Two objects were investigated - a DC electric motor and a scale model of the airframe made by 3D printing. A generalized diagram of the experimental setup is shown in Figure 7. In Figure 7 we use following denotations: DUT - Device Under Test, FVS - Fiber Vibration Sensor, EVS - Electrical Vibration Sensor, OSC with FFT - Osciloscope with Fast Fourier Transform function analysis.

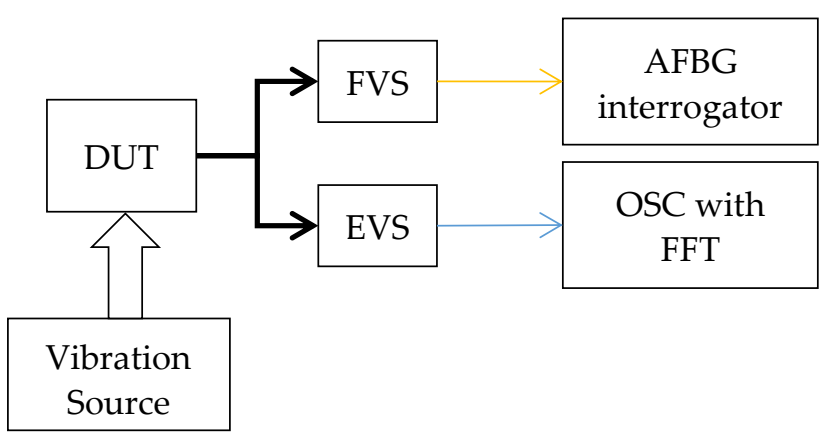

Figure 7. Block diagram of the experimental setup: DUT - Device Under Test, FVS - Fiber Vibration Sensor, based on AFBG, EVS - Electrical Vibration Sensor (ADXL203CE), OSC with FFT Osciloscope with Fast Fourier Transform function analysis (DSO 7104 from Agilent Technologies).

Here are some of the metrological characteristics of the equipment used.

Table 1. Metrological characteristics.

\begin{tabular}{cc}
\hline Device & Paramter: value, units \\
\hline & Dynamic range: $0 . . \pm 5 \mathrm{G}$ \\
ADXL203CE & Bandwidth: $2.5 \mathrm{kHz}$ \\
& Output signal scale: $1000 \mathrm{mv} / \mathrm{G}$ \\
& Output noise: $0.4 \mathrm{mv} \mathrm{rms}$ \\
\hline & Bandwidth: $1 \mathrm{GHz}$ \\
DSO 7104B & Sample rate: $4 \mathrm{Gsmpl} / \mathrm{s}$ \\
& ADC: 8 bit \\
\hline
\end{tabular}

FVS

Address frequency: $\sim 6,25 \mathrm{GHz}$

Address bandwidth: $\sim 400 \mathrm{MHz}$

Sensitivity: $\sim 0.95 \mathrm{pm} / \mu \varepsilon$ 


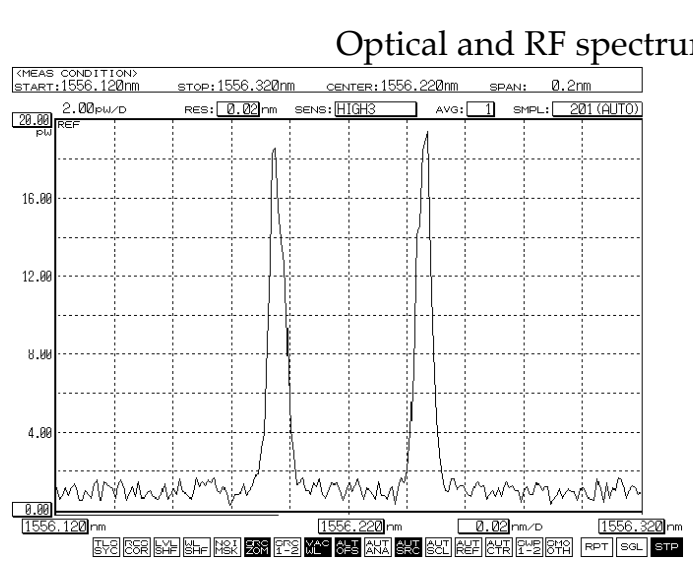

(a)

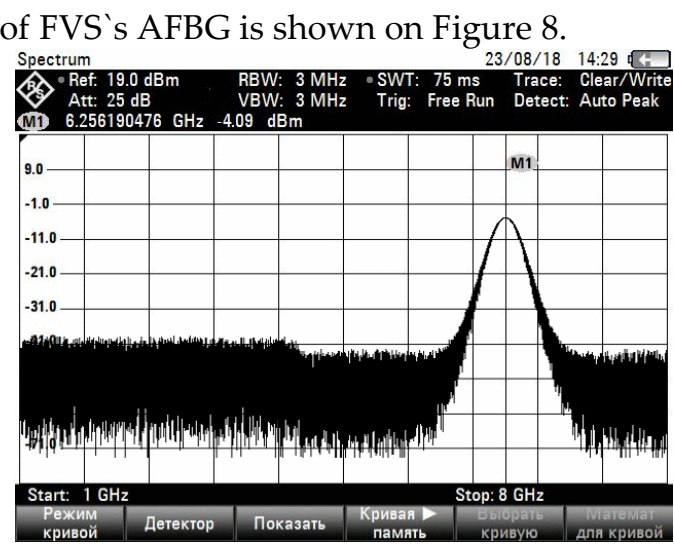

(b)

Figure 8. Optical spectrum of the central (addressing) part of AFBG (a) and radio frequency spectrum at the address frequency (b).

In order to exclude the influence of the design of the optical sensor on the measurements, in both cases, the AFBG glued to the object of research acted as FVS. As a glue, we used a specialized adhesive for strain gauge resistors M-Bond 610. This approach, in addition, will allow in the future to use AFBGs embedded directly into the structural elements of the object.

\subsection{Motor Study}

A low-power DC motor was used as the object under study in the first series of experiments. In Figure 9 shows the placement of the FVS and the EVS board on the motor housing.

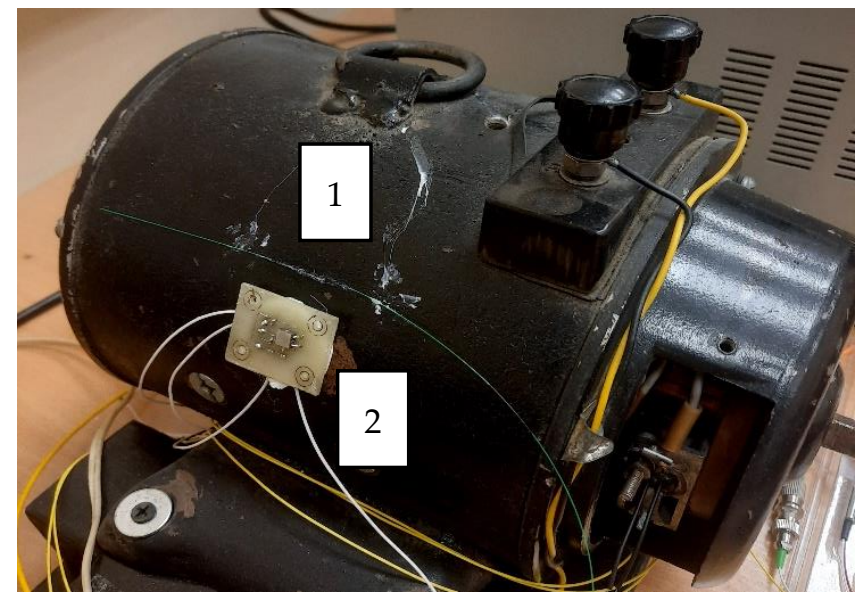

Figure 9. Motor under study with FVS (1) and EVS (2) placed.

Two series of experiments were carried out: the spectra of natural vibrations of an operating electric motor at a fixed rotation speed with a balanced and unbalanced (defective) shaft were constructed. Below are the spectrograms captured by FVS and EVS (Figures 10 and 11). 


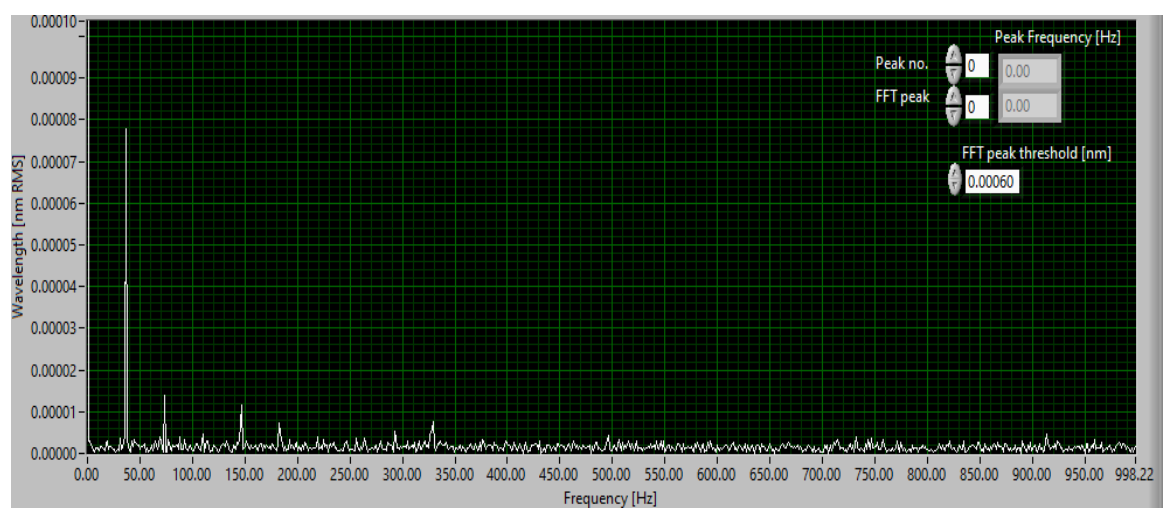

(a)

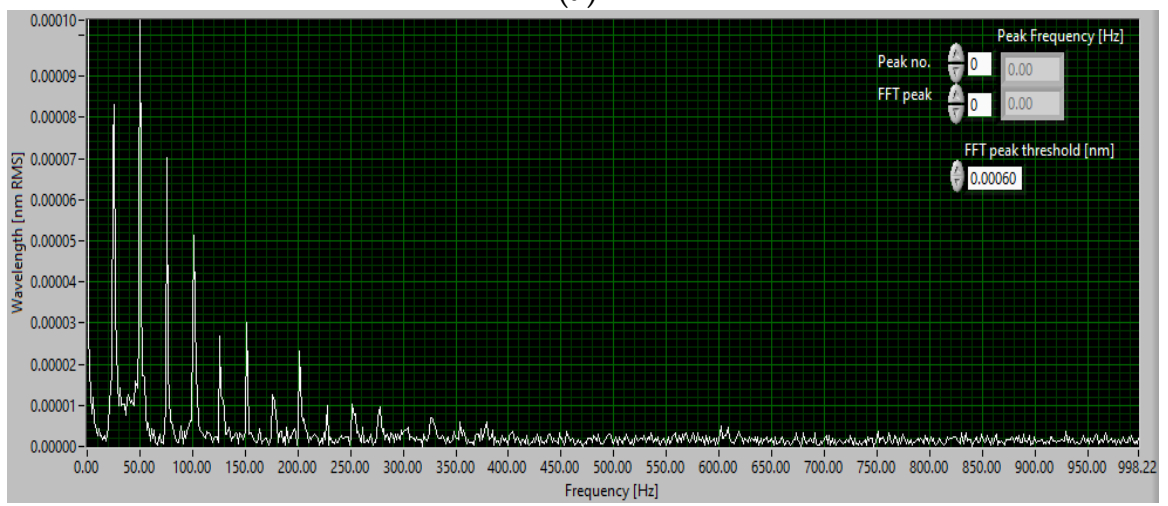

(c)

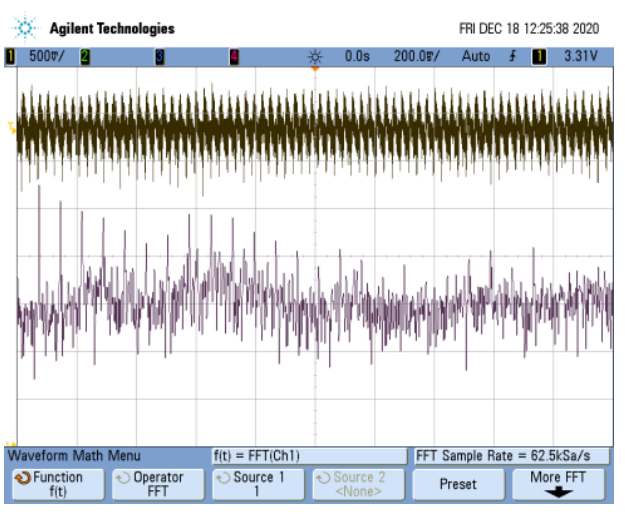

(b)

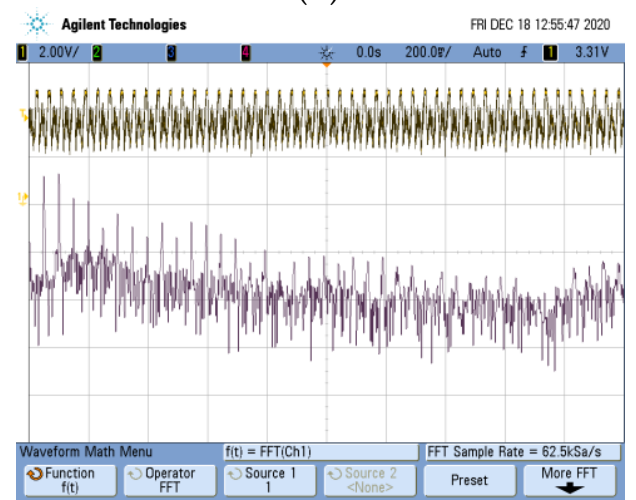

(d)

Figure 10. Motor vibration spectrum: FVS (a) and EVS (b) readings for a balanced shaft; readings FVS (c) and EVS (d) for an unbalanced shaft. The grid step for (b) and (d) is $100 \mathrm{~Hz}$.

Analyzing the data obtained, the following conclusions can be drawn. In both cases, the EVS spectrum contains a greater number of harmonics, which can be explained, probably, by the poor fixing of the sensor to the housing. The SNR of the fundamental harmonic for FVS is at least 16 times higher ( $\operatorname{minSNR}$ FVS $=40$, SNREvS $=2.5$ ). The FVS spectrogram is "cleaner" - the fundamental $40 \mathrm{~Hz}$ harmonic is clearly visible, and in the case of an unbalanced shaft, its amplitude is much less but still noticeable; in addition, it was noticed that even in the absence of vibration, the output spectrum of the EVS will have a harmonic of $50 \mathrm{~Hz}$ (mains frequency) when the microcircuit is powered from a laboratory power source, in order to eliminate this circumstance during the experiments, the microcircuit was powered from a galvanic source.

\subsection{Airframe Model Study}

For this experiment, a model of the airframe of the An-124 "Ruslan" aircraft was made by 3D printing. FVS were fixed on the wing consoles (see Figure 11) from the upper side, and EVS - from the bottom (not shown in the figure). 


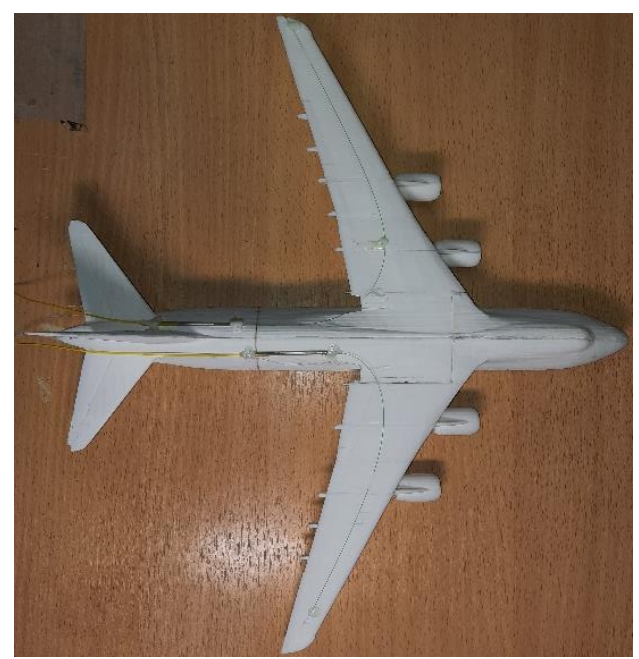

Figure 11. Airplane glider model with placed FVS.

Similar to the previous experiment, spectrograms of the forced vibrations of an object (frequency of action $100 \mathrm{~Hz}$, amplitude 2G) with a correct and defective wing were obtained (Figure 12).

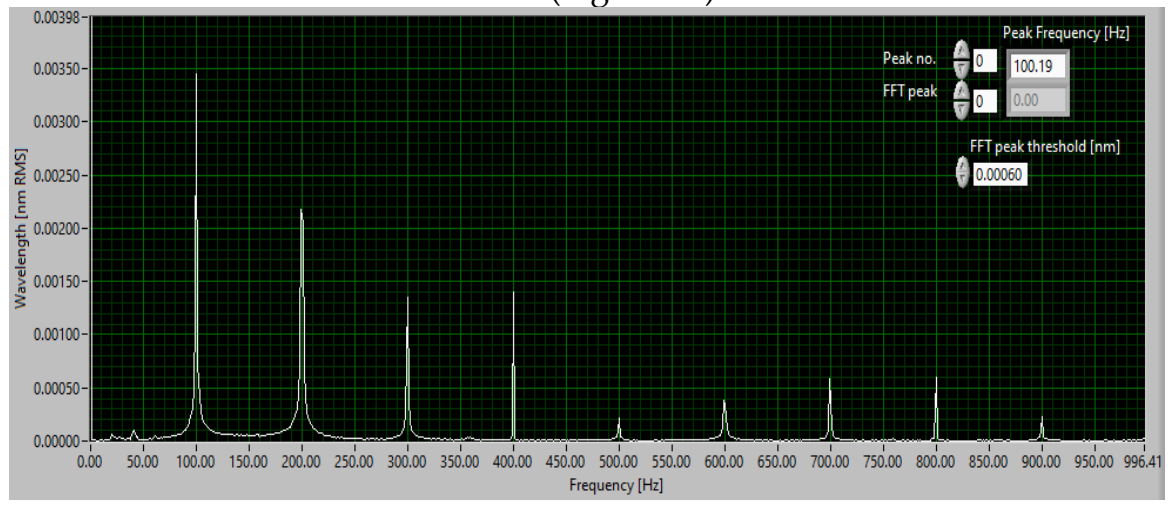

(a)

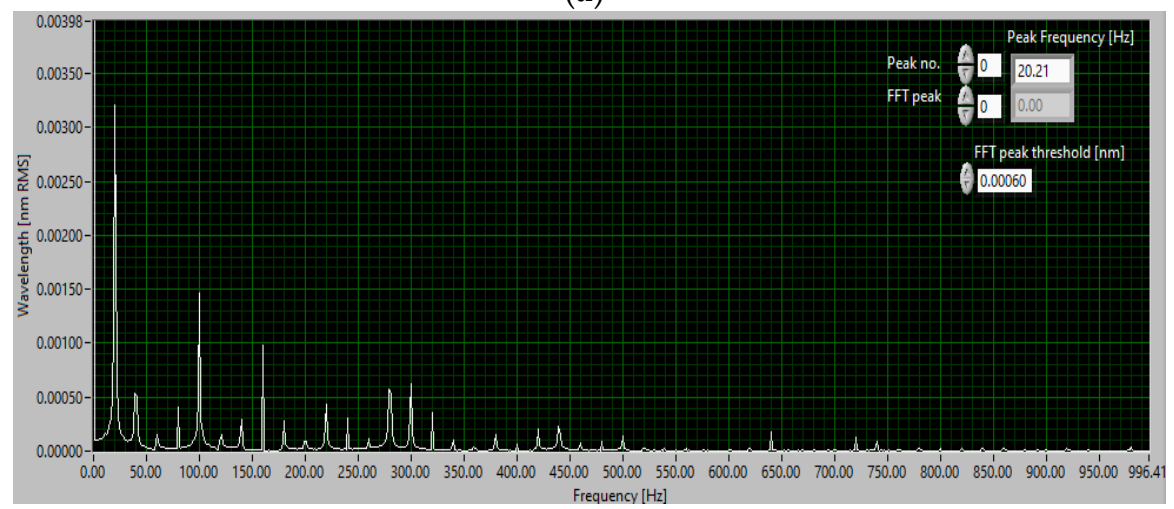

(c)

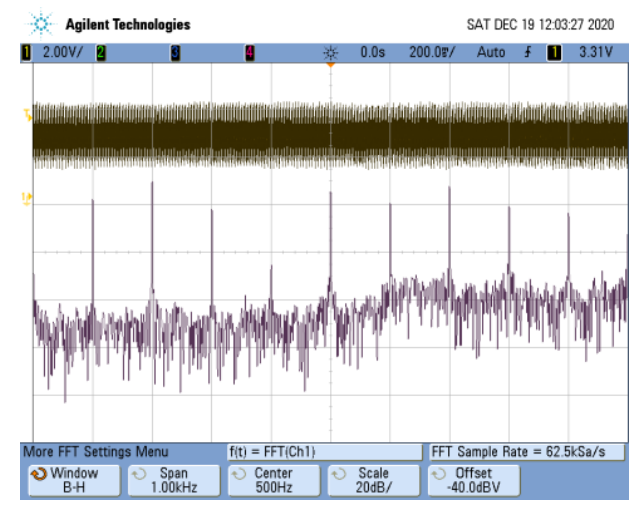

(b)

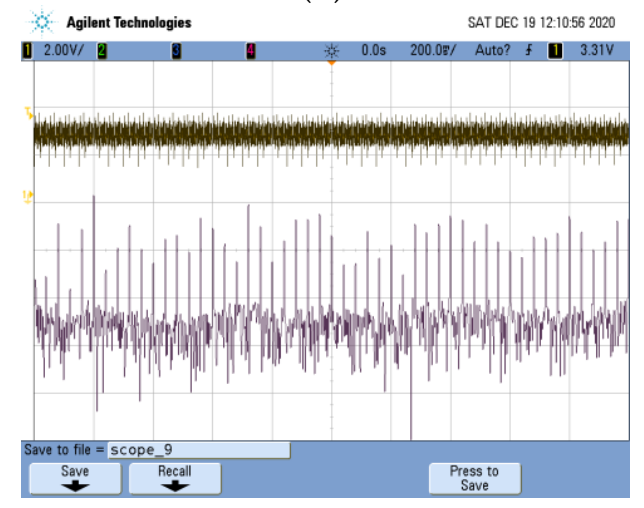

(d)

Figure 12. The spectrum of the forced vibrations of the airframe of the aircraft: FVS (a) and EVS (b) readings for the right wing; FVS (c) and EVS (d) readings for the defective wing. Grid step for (b) and $(\mathrm{d})-100 \mathrm{~Hz}$.

The general conclusions are similar to the previous experiment: FVS has a higher SNR; for a serviceable wing, the spectra obtained by both sensors are completely identical; for a defective wing, a larger number of harmonics are present in the EVS spectrum, which is probably due to the interaction of structural elements of the board with the sensor and the object under study.

Thus, both experiments confirmed that the rejection of the mechanical elements of the sensor and the placement of the sensitive element directly on the controlled object 
increases the reliability of the measurements, especially with a large vibration amplitude, while no tangible loss of sensitivity was observed - SNR for FVS in both cases was much higher.

It should be noted that similar results can be obtained using FVS based on conventional FBGs, however, existing interrogators limit the controlled frequency band to units of kilohertz. The use of AFBG makes it possible to significantly expand this band (for example, to hundreds of kilohertz), and direct placement of the AFBG on the controlled object will exclude the influence of the mechanical transducer of a traditional sensor on the band and dynamics of the measured vibrations. In addition, the AFBG interrogator does not contain tunable optical elements or volumetric optics, which potentially simplifies its use on mobile objects with difficult operating conditions.

\section{Conclusions}

The paper presents a new approach to using AFBG for vibration control in a wide frequency band. A mathematical model of the measuring conversion was developed and investigated, an experimental study of the FVS based on AFBG was carried out, which showed a noticeable (at least an order of magnitude) gain in SNR compared to EVS and higher measurement reliability due to the rejection of the mechanical sensor transducer and the placement of the sensitive element directly on the structural elements of the controlled object.

Further research will be devoted to the construction of measuring systems based on FVS arrays on AFBG, as well as the experimental study of vibrations in a wider (US) frequency band.

Author Contributions: Conceptualization, A.K. and O.M.; methodology, A.S.; software, K.K.; validation, A.K.; formal analysis, A.K.; investigation, A.S.; resources, K.L.; data curation, K.K.; writingoriginal draft preparation, K.L.; writing - review and editing, A.K.; visualization, K.L.; supervision, O.M.; project administration, O.M.; funding acquisition, K.L. All authors have read and agreed to the published version of the manuscript

Funding: A.S. and O.M. were funded by the Ministry of Science and Higher Education of the Russian Federation (Agreement No. 075-03-2020-051, Topic No. fzsu-2020-0020), A.K. and K.L. were funded by a grant from the President of the Russian Federation for state support of young Russian scientists - candidates of sciences MK-3421.2019.8 (Agreement No. 075-15-2019-309).

Conflicts of Interest: The authors declare no conflict of interest.

\section{References}

1. Garc'1a, Y. R.; Corres, J. M.; Goicoechea, J. Vibration Detection Using Optical Fiber Sensors. Journal of Sensors 2010, Volume 2010, $12 \mathrm{p}$.

2. Giallorenzi, T. G.; Bucaro, J. A.; Dandridge A. Optical fiber sensor technology. IEEE Journal of Quantum Electronics 1982, Volume 18, no. 4, pp. 626-665.

3. Vento, J. R.; Hernandez, L. 1.; A. Santamarı, Perez-Jimenez, R.; Rabadan, J. A. Infrared wireless DSSS system for indoor data communication links. Optical Wireless Communications II 1999, Volume 3850 of Proceedings of the SPIE, pp. 92-99.

4. Hongbin, R.; Jinlei, J.; Zhishe, C.; Dengyu, Z. Application of Spread Spectrum Technology to Measurement, Air Force Institute of Missile, Shaanxi, China, 1997.

5. Perrone, G.; Vallan, A. A low-cost optical sensor for noncontact vibration measurements. IEEE Transactions on Instrumentation and Measurement 2009, Volume 58, no. 5, pp. 1650- 1656.

6. Culshaw B. Optical Fibre Sensing and Signal Processing; P. Pergrinus, London, UK, 1984.

7. Aydin, N.; Arslan, T.; Cumming, D. R. S. A direct-sequence spread-spectrum communication system for integrated sensor microsystems. IEEE Transactions on Information Technology in Biomedicine 2005, Volume 9, no. 1, pp. 4-12.

8. Pitt, G. D.; Extance, P.; Neat, R. C. Optical-fibre sensors. IETE Technical Review 1986, Volume 3, no. 8, pp. $379-417$.

9. Gunasilan, U. Operative factors contributing to the selection of fiber-optic techniques for remote measurement of strain/stress. In Proceedings of the IEEE 9th International Conference on Computer and Information Technology, Xiamen, China, 11-14 Oct. 2009.

10. Hecht, J. Understanding Fiber Optics; Pearson Prentice Hall, NJ, USA, 2006.

11. Ione, S.D.; Limanova, N.I. Fiber-optic sensor. Patent RU № 2267085, 27.12.2005.

12. Gangopadhyay, T. K.; Chakravorti, S.; Chatterjee, S.; Bhattacharya, K. Multiple fringe and nonsinusoidal signals obtained from a fiber-optic vibration sensor using an extrinsic Fabry-Perot interferometer. Measurement Science and Technology 2005, Volume 16, pp. 1075-1082. 
13. Zhang, X.; Liu, X.; Zhang, F.; Sun, Zh.; Min, L.; Li, Sh. Reliable high sensitivity FBG geophone for low frequency seismic acquisition. Measurement 2018, Volume 129, pp. 62-67.

14. Zhang, X.; Rong, Q.; Sun, H.; Yang, Sh.; Yuan, L.; Hu, M. Low-frequency fiber Bragg grating accelerometer based on a doublesemicircle cantilever. Optical Fiber Technology 2014, Volume 20, pp. 190-193.

15. Zhang, Y.; Qiao, X.; Liu, Q.; Yu, D.; Gao, H.; Shao, M. Study on a fiber Bragg grating accelerometer based on compliant cylinder. Optical Fiber Technology 2015, Volume 26, pp. 229-233.

16. Weng, Y.; Qiao, X.; Guo, T.; Hu, M.; Feng, Zh.; Wang, R. A Robust and compact Fiber Bragg Grating vibration sensor for seismic measurement. IEEE Sensors journal 2011, Volume 12, no. 4, pp. 800-804.

17. Weng, X.; Guo, Y.; Xiong, L.; Wu, H. High Frequency Optical Fiber Bragg Grating Accelerometer. IEEE Sensors journal 2018, Volume 18, no. 12, pp.4954-4960.

18. Lipatnikov, K.A.; Sahabutdinov, A. Zh.; Nureev, I.I.; Kuznetsov, A.A.; Fashutdinov, L.M. Fiber-Optic Vibration Sensor «VIBA». In 2019 Systems of Signals Generating and Processing in the Field of on Board Communications, Moscow, Russia, 20-21 March 2019, IEEE.

19. Burgmeier, J.; Koch, J.; Wolfgang, Sc.; Intensity-independent fiber coupled interrogation technique for fiber Bragg gratings by fiber Bragg gratings. Proc. of SPIE 2012, Volume 8421, pp. 84215G-2

20. Morozov, O.; Sakhabutdinov, A.; Anfinogentov, V.; Misbakhov, R.; Kuznetsov, A.; Agliullin, T. Multi-Addressed Fiber Bragg Structures for Microwave-Photonic Sensor Systems. Sensors 2020, Volume 20, 2693.

21. Agliullin, T.; Gubaidullin, R.; Sakhabutdinov, A.; Morozov, O.; Kuznetsov, A.; Ivanov, V. Addressed Fiber Bragg Structures in Load-Sensing Wheel Hub Bearings. Sensors 2020, Volume 20, 6191.

22. Morozov OG, Sakhabutdinov AJ. Addressed fiber Bragg structures in quasidistributed microwave-photonic sensor systems. Computer Optics 2019; 43(4): 535-543. DOI: 10.18287/2412-6179-2019-43-4-535-543. 